Case report

\title{
An unusual case of severe myocardial dysfunction in a patient with giant left atrium and frequent right ventricular pacing
}

\author{
Andrey Bobrov ${ }^{1}$, Alexey Tsyganov ${ }^{2}$, Anton Barsukov ${ }^{1}$, Maria Mirokhina ${ }^{1}$ \\ ${ }^{1}$ Military Medical Academy, Saint Petersburg, Russia \\ ${ }^{2}$ Petrovsky National Research Centre of Surgery, Moscow, Russia
}

Received 26 September 2016, Accepted 25 October 2016

(C) 2016, Bobrov A., Tsyganov A., Barsukov A., Mirokhina M.

C 2016, Russian Open Medical Journal

Abstract: Giant left atrium is a rare condition, with a reported incidence of $0.3 \%$, and mainly associated with rheumatic mitral valve disease with predominant regurgitation. Giant left atrium has significant hemodynamic effects and requires specific management in the most of the cases. We present an unusual case of a 65-year-old woman with massive left atrial enlargement accompanied by frequent right ventricular pacing, who was documented with severe pacing-induced left ventricular dyssynchrony.

Keywords: giant left atrium, ventricular pacing, strain analysis, ventricular dysfunction, heart failure.

Cite as Bobrov A, Tsyganov A, Barsukov A, Mirokhina M. An unusual case of severe myocardial dysfunction in a patient with giant left atrium and frequent right ventricular pacing. Russian Open Medical Journal 2016; 5: e0407.

Correspondence to Andrey Bobrov. Address: 6, Academika Lebedeva str., Saint Petersburg, 194044, Russia. Tel.: +7 9213704869.

E-mail: andreybobrov@me.com

\section{Introduction}

Giant left atrium (GLA) is a rare condition, with a reported incidence of $0.3 \%$, and mainly associated with rheumatic mitral valve disease with predominant regurgitation. The majority of patients are symptomatic with the most common symptoms being that of shortness of breath, dysphagia, palpitations, chest pain, and thromboembolic events [1]. However, other reasons for clinical deterioration of heart failure in massive enlargement of the left atrium are a rare entity. In this case, we discuss etiology, clinical presentation, and management modalities in unusual case of severe myocardial dysfunction in a patient with GLA and frequent right ventricular (RV) pacing after heart valve surgery and pacemaker implantation.

\section{Case report}

We present the case of a 65 -year-old female with rheumatic heart disease, initially diagnosed at 22 years of age. In 1972, she underwent commissurotomy of mitral valve due to mitral stenosis, then in 1997, she underwent redo operation with mitral valve replacement using mechanical valve. Two years ago single chamber pacemaker was implanted because of transient atrioventricular block. The leading clinical problem was shortness of breath on minimal exertion in the last month. When the patient was presented to our outpatient clinic for the first time, acrocyanosis, swollen feet, hepatomegaly, and hypotension (systolic and diastolic blood pressures were 90 and $60 \mathrm{mmHg}$ ) were noted. Electrocardiogram (ECG) revealed atrial fibrillation at rates of 60 to $65 \mathrm{bpm}$ with frequent episodes of RV pacing at rate of $60 \mathrm{bpm}$ (Figure 1, panel A). Chest X-ray revealed severe cardiomegaly and splaying of the carina (Figure 1, panel B). Echocardiography revealed GLA (anterior-posterior diameter $11.4 \mathrm{~cm}$ ), severe systolic and diastolic left ventricular (LV) dysfunction (ejection fraction (EF) $-15 \%, E / e^{\prime}$ ratio -17 ) as well as severe LV enlargement (end-diastolic volume (EDV) - $280 \mathrm{ml}$ ), normal volumes of right cardiac chambers, and no pulmonary hypertension (Figure 1, panels C and D; Supplementary Videos 1 and 2). There were no any signs of prosthetic mitral valve dysfunction. Septal to posterior wall delay during RV pacing was increased to $146 \mathrm{~ms}$ (Figure 2, panel A). Signs of severe intraventricular dyssynchrony during 2D-strain analysis were detected (Figure 2, panel C). Multislice computed tomography of the heart excluded coronary artery disease and proved GLA. Volume of the left atrium was $1398 \mathrm{ml}$ (Figure 3). Cumulative percent of RV pacing was $64 \%$ determined from stored pacemaker data. We supposed decreasing pacemaker lower rate limit to 40 bpm could reduce ventricular desynchronisation and improve global myocardial contractility, even through presence of the GLA and severe LV dysfunction. Reexamination after 3 months showed reducing of breathlessness, improving LV function (EF $-31 \%, E / e^{\prime}$ ratio - 15), and dramatic decreasing cumulative percent of RV pacing (1\%). Septal to posterior wall delay was decreased to $4 \mathrm{~ms}$ (Figure 2, panel B) and intraventricular dyssynchrony disappeared (Figure 2, panel D). 

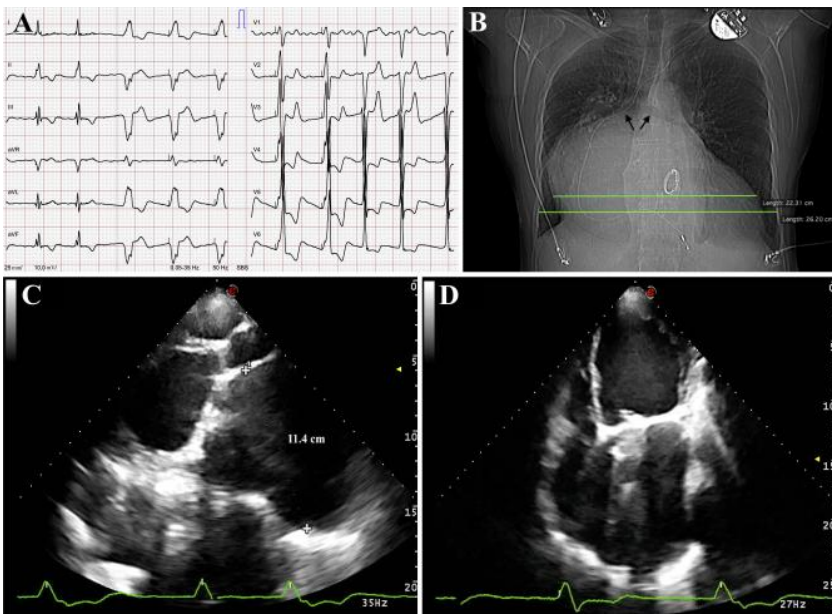

Figure 1. 12-lead ECG showing atrial fibrillation with frequent episodes of ventricular pacing (panel $A$ ). Chest $x$-ray showing massive cardiomegaly with a cardiothoracic ratio of 0.85 , splaying of the carina (arrows), a mitral valve prosthesis, and pacemaker (panel B). Echocardiography showing the giant left atrium and enlarged left ventricle. Modified parasternal long axis (panel C) and apex 4-chamber views (panel D).

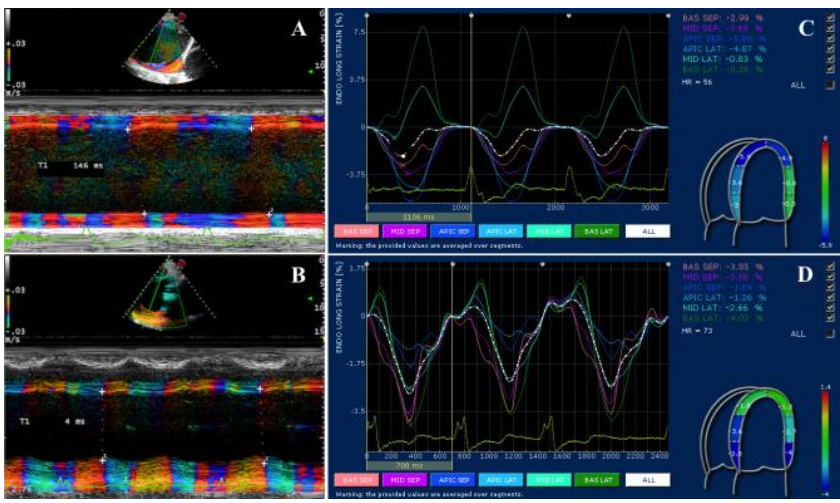

Figure 2. Color tissue M-mode echocardiography in parasternal long axis view showing value of septal to posterior wall delay at the initial evaluation (panel A) and after 3 months (panel B). Apex 4-chambers view of 2D strain echocardiography showing segmental values of longitudinal myocardial strain at the initial evaluation (panel C) and after 3 months (panel D).
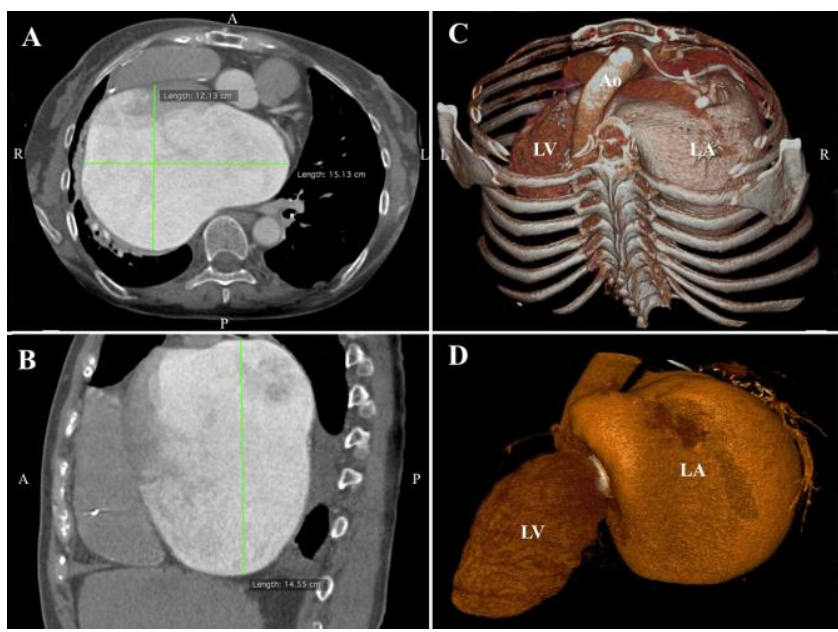

Figure 3. Computed axial tomography showing massive left atrial enlargement: $15.1 \times 12.1 \times 14.6 \mathrm{~cm}$ (panel $A$ and $B$ ). A volume-rendering computed tomography scans demonstrating the giant left atrium (panel $\mathrm{C}$ and D).

\section{Discussion}

GLA is a rare entity in the current era. GLA is defined as a cardio-thoracic ratio $>0.7$ combined with a left atrial anteriorposterior diameter of $>8 \mathrm{~cm}$ on transthoracic echocardi-ography and typically found in patients who have rheumatic mitral valve disease [2]. The cause of GLA remains unknown, but since it is so strongly associated with rheumatic heart disease, there is hypothesis that rheumatic pancarditis damages the entire heart, including the atria [3]. It is closely related to rheumatic mitral regurgitation or mixed mitral valve disease with predominant regurgitation, but can occur in patients with rheumatic mitral stenosis or mitral regurgitation due to other causes [4-6]. Moreover, isolated reports in the literature of patients with GLA and mitral valve prosthesis sustain that atrial enlargement also affected by the quality of the left atrial wall [7]. Atrial fibrillation, which can occur secondary to left atrial enlargement, contributes more to the increase in overload on the left atrium and causes more enlargements.

Patients usually presents with complaints of shortness of breath. Additionally, GLA can cause intracardiac or extracardiac compression manifestations and thromboembolic events. In our patient, there was no available data about her condition before the mitral valve replacement, which had been performed 19 years ago. She had no symptoms of compression and the dyspnea was her main complaint upon admission. The low-output syndrome and respiratory failure were first thought to be due to the GLA. The volume reduction surgery results in a significant decrease in the incidence of low-output syndrome and respiratory failure, but indications are controversial. The main indication for volume reduction, which generally surgery performed in addition to mitral valve surgery, is the presence of intracardiac or extracardiac compressive symptoms or presence of thrombus [8]. In this case patient had no any signs of compressive symptoms, prosthetic mitral valve dysfunction, presence of thrombus or a history of thromboembolic events as well as risk of isolated volume reduction was estimated as very high.

In other hand in pacemaker patients, left ventricular dysfunction can occur due to chronic RV apical pacing [9]. Upgrading to biventricular pacing is an option to reverse LV dysfunction but reprogramming of the pacemaker can also be favorable [10]. Echocardiography demonstrated a tremendous improvement in LV function during 3 months after reprogramming of the pacemaker, whereas the percentage of RV pacing diminished dramatically. Frequent RV pacing was associated with rapid worsening of heart failure in patient with initial severe left cardiac chambers enlargement due to rheumatic heart disease and concomitant mechanical LV dyssynchrony. Careful analysis of the cause of LV deterioration precluded unnecessary surgery or upgrading to biventricular pacing.

\section{Conclusion}

Pacemaker patients following cardiac surgery requiring bradycardia pacing should be cared with particular attention to clinical worsening, not only because of possible prosthetic valve dysfunction or rare heart condition, but also because of pacinginduced LV dyssynchrony. Moreover, we must be alert to the fact that severe primary left atrial disease can produce GLA even in absence of predominant mitral regurgitation. 


\section{Supplementary files}

Video 1. Echocardiography showing the giant left atrium and enlarged left ventricle. Apex 4-chamber view.

URL: https://youtu.be/7EFhJGa4l_8

Video 2. Echocardiography showing the giant left atrium and enlarged left ventricle. Parasternal long axis view.

URL: https://youtu.be/P_E-VIc3JYc

\section{Acknowledgement}

We thank Igor Zheleznyak and Igor Menkov for their help with computed tomography data.

\section{Conflict of interest}

The authors declare no conflict of interest.

\section{References}

1. El Maghraby A, Hajar R. Giant Left Atrium: A Review. Heart Views 2012; 13: 46-52. PMID: 22919448. DOI: 10.4103/1995-705X.99227.

2. Piccoli GP, Massini C, Di Eusanio G, Ballerini L, lacobone G, Soro A, Palminiello A. Giant left atrium and mitral valve disease: Early and late results of surgical treatment in 40 cases. J Cardiovasc Surg 1984; 25: 328-336. PMID: 6237112.

3. Hurst W. Memories of patients with a giant left atrium. Circulation 2001; 104: 2630-2631. PMID: 11723007. DOI: 10.1161/hc4701.100775.

4. Ates $M$, Sensoz $Y$, Abay $G$, Akcar M. Giant left atrium with rheumatic mitral stenosis. Tex Heart Inst J 2006; 33: 389-391. PMID: 17041705.

5. Ahmed I, Sarkar A, Pande A, Kundu C. A "non-rheumatic" giant left atrium. Ann Pediatr Cardiol 2015; 8: 95-97. PMID: 25684900. DOI: 10.4103/0974-2069.149542.

6. Yuksel UC, Kursaklioglu H, Celik T. Apical hypertrophic cardiomyopathy with giant left atrium. Arq Bras Cardiol 2007; 88: e47. PMID: 17384827. DOI: $10.1590 /$ S0066-782X2007000200024.

7. Celik T, Bozlar U, Celik M, Demirkol S, Iyisoy A, Cingoz F. Giant left atrium in a patient with Starr-Edwards caged ball implanted three decades ago. Cardiovasc Revasc Med 2011; 12: 266-267. PMID: 21130710. DOI: 10.1016/j.carrev.2010.09.004.

8. Apostolakis E, Shuhaiber JH. The surgical management of giant left atrium. Eur J Cardiothorac Surg 2008; 33: 182-190. PMID: 18096399. DOI: $10.1016 /$ j.ejcts.2007.11.003.

9. Tops LF, Schalij MJ, Bax JJ. The effects of right ventricular apical pacing on ventricular function and dyssynchrony implications for therapy. J Am Coll Cardiol 2009; 54: 764-776. PMID: 19695453. DOI: 10.1016/j.jacc.2009.06.006.

10. den Besten T, Sedney MI, Frederiks J, van Hemel NM. Left ventricular dysfunction due to right ventricular stimulation: is biventricular upgrade really necessary? Neth Heart J 2010; 18: 604-605. PMID: 21301623. DOI: $10.1007 / \mathrm{s} 12471-010-0842-\mathrm{x}$.

\section{Authors:}

Andrey Bobrov - MD, PhD, Head of Cardiac Diagnostic Unit, Assistant Professor, Internal Medicine Clinic, Military Medical Academy, Saint Petersburg, Russia.

Alexey Tsyganov - MD, Head of Cardiac Electrophysiology Department, Petrovsky National Research Centre of Surgery, Moscow, Russia.

Anton Barsukov - MD, PhD, Deputy Head of Internal Medicine Clinic, Professor, Military Medical Academy, Saint Petersburg, Russia.

Maria Mirokhina - MD, PhD, Cardiologist, Internal Medicine Clinic, Military

Medical Academy, Saint Petersburg, Russia. 